\title{
Quasars, Feedback, and Galaxy Formation
}

\author{
Philip F. Hopkins \\ Department of Astronomy, University of California Berkeley, Berkeley, CA 94720, USA \\ Email: phopkins@astro.berkeley.edu
}

\begin{abstract}
Recent observations of tight correlations between supermassive black hole masses and the properties of their host galaxies demonstrate that black holes and bulges are co-eval and have motivated theoretical models in which feedback from AGN activity regulates the black hole and host galaxy evolution. Combining simulations, analytic models, and recent observations, answers to a number of questions are starting to take shape: how do AGN get triggered? How long do they live? What are typical light curves and what sets them? Is feedback necessary and/or sufficient to regulate BH growth? What effects does that feedback have on the host galaxy? On the host halo? All of this also highlights questions that remain wide open: how does gas get from a few pc to the AGN? What are the actual microphysical mechanisms of feedback? What is the tradeoff between stellar and AGN feedback? And, if there are different "modes" of feedback, where/when are each important?
\end{abstract}

Keywords. (galaxies:) quasars: general, galaxies: active, galaxies: nuclei, galaxies: evolution, galaxies: formation, galaxies: Seyfert, cosmology: theory

\section{Introduction}

Observations have shown that the masses of supermassive black holes (BHs) correlate with various host galaxy bulge properties including mass (Magorrian et al. 1998), velocity dispersion (Ferrarese \& Merritt 2000; Gebhardt et al. 2000), concentration and/or light profile (Graham et al. 2001), and spheroid potential well depth/binding energy (Hopkins et al. 2007b; Aller \& Richstone 2007). These correlations exhibit little scatter, despite the fact that, for example, the stellar mass, light profile, and other properties at the BH radius of influence scatter by an order of magnitude at fixed galaxy velocity dispersion or mass (Hopkins et al. 2009b). Together with constraints indicating that most of the $\mathrm{BH}$ mass is assembled in optically bright quasar phases (Soltan 1982; Salucci et al. 1999; Yu \& Tremaine 2002; Hopkins et al. 2006a), this has led to the development of models where large-scale effects of feedback processes from accretion self-regulate $\mathrm{BH}$ growth at a critical mass (Silk \& Rees 1998; King 2003; Di Matteo et al. 2005; Murray et al. 2005). Gas inflows triggered by some process fuel rapid BH growth until feedback begins to expel nearby gas and dust. This "blowout" results in a short-lived, bright optical quasar that, having expelled its fuel supply, fades and leaves a remnant on the observed BH-host correlations (Hopkins et al. 2005a,c). These scenarios have been able to explain many quasar observables, including luminosity functions, lifetimes, and $\mathrm{BH}$ mass functions (Hopkins et al. 2005b, 2006b, 2008, 2009a; Volonteri et al. 2006; Menci et al. 2003; Somerville et al. 2008; Lapi et al. 2006; Tortora et al. 2009).

But the actual mechanisms of feedback and ISM physics remain highly uncertain, and observational tests of these scenarios are needed to constrain the behavior of outflows on galactic scales. In the simulations of Springel et al. (2005), BH feedback is coupled in a simple, energetic fashion (a fraction of the accretion energy is deposited in gas on the smallest scales resolved). There is some motivation for this: highly energetic outflows are associated with bright quasars (for a review, see Veilleux et al. 2005); these range 
from intense winds $\left(v \sim 10^{4} \mathrm{~km} \mathrm{~s}^{-1}\right)$ associated with the central engine seen in the broad emission-line regions and broad absorption-line quasars (Weymann et al. 1981) to more moderate outflows $\left(v \sim 10^{2}-10^{3} \mathrm{~km} \mathrm{~s}^{-1}\right)$ associated with the narrow-line region and the "warm absorber" (Laor et al. 1997; Crenshaw et al. 2000) as well as with small-scale quasar absorption and occultation systems (e.g., McKernan \& Yaqoob 1998; Turner et al. 2008; Miller et al. 2008). Indeed, high-velocity winds driven near the accretion disk and Compton heating of low-density gas are theoretically hard to avoid (see, e.g., Blandford \& Payne 1982; Begelman 1985; Konigl \& Kartje 1994; Elvis 2000; Sazonov et al. 2005; Proga 2000, 2007). However, the mass flux in these winds is difficult to determine it may be small $\left(\lesssim \dot{M}_{\mathrm{BH}}\right)$, although at least in some cases the outflowing material is extremely dense (Hall \& Hutsemèkers 2003; Hall et al. 2007), and their effect on the medium will non-trivially depend on its phase structure and other properties (Hopkins \& Elvis 2009).

\section{Overview}

A large number of models have attempted to include simple prescriptions for AGN feedback in simulations, in order to reproduce observables such as the $M_{\mathrm{BH}}-\sigma$ relationship. The basic argument is as follows: if the $\mathrm{BH}$ is accreting near the Eddington limit $\left(L_{\mathrm{bol}} \propto M_{\mathrm{BH}}\right)$ and some fraction (typically a few percent) of the AGN radiant energy can couple to the surrounding material, it will be comparable to the binding energy of the gas in the galaxy when the $\mathrm{BH}$ reaches some critical limit at about $10^{-3} M_{\text {gal }}$. In a series of papers, Springel et al. (2005), Di Matteo et al. (2005), Hopkins et al. (2005d), and Hopkins et al. (2005a) have described the effects of a simple implementation of

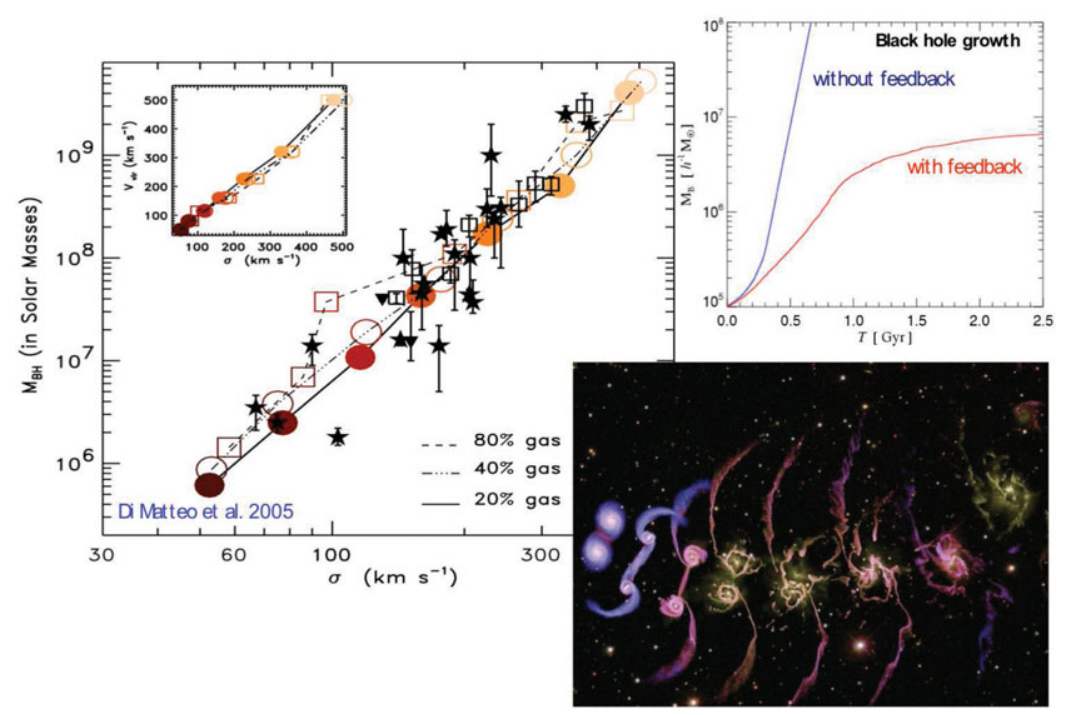

Figure 1. The BH-host galaxy relation as produced by a simple model where $5 \%$ of the radiated $\mathrm{BH}$ accretion energy couples to the surrounding gas as thermal energy, in Di Matteo et al. (2005). Left: Resulting $M_{\mathrm{BH}}-\sigma$ relation. Points and lines show simulation results, points with error bars show the observed galaxies compiled by Tremaine et al. (2002). Inset shows the relation between $\sigma$ and host halo circular velocity. Top Right: BH mass versus time in a simulation with and without feedback. Bottom Right: Image of the gas surface density (brighter is higher density) in such a simulation at different times, where the "blowout" induced by feedback appears midway through the sequence shown. 
such a model. Specifically, in simulations of violent, gas-rich events (say a galaxy-galaxy merger), the $\mathrm{BH}$ is assumed to accrete at the Bondi-Hoyle rate estimated from gas at the resolution limit of the simulation $(\sim 50-100 \mathrm{pc})$. If a radiative efficiency of $10 \%$ is assumed, then $5 \%$ of the implied luminosity is returned to the nearby gas within the central smoothing kernel around the BH in the form of thermal energy. Figure 1 shows the results from such a model, as first shown by Di Matteo et al. (2005). Without feedback, the $\mathrm{BH}$ would accrete until the nuclear gas supply is entirely exhausted, overshooting the observed $\mathrm{BH}$-host relations by a large margin. With feedback present, the $\mathrm{BH}$ reaches a critical luminosity (accreting near the Eddington limit, so this equates to a critical mass), and then drives a "blowout," where nuclear gas is expelled, leaving a remnant in good agreement with the observed $M_{\mathrm{BH}}-\sigma$ relation.

\section{Implications}

This confirms the expectations of a simple "back of the envelope" calculation for the ability of a simple feedback to explain the observed relationship. But does it make unique predictions? Other than the feedback winds themselves, which may be extremely difficult to directly observe, there are in fact a number of important impliciations. Figure 2 illustrates one of these. Younger et al. (2008) use the model of Di Matteo et al. (2005) to make predictions for the $\mathrm{BH}$-host galaxy correlations that result from galaxy-galaxy minor mergers and $\mathrm{BH}$ fueling from isolated disk instabilities (e.g., galaxy bars). They find qualitatively similar correlations arise, and similar results are seen by Johansson et al. $(2009 \mathrm{a}, \mathrm{b})$. But the key parameter that dictates the BH mass, in any model qualitatively similar to the one described above, is not the galaxy mass or dispersion specifically, but the binding energy of the gas at the center of the galaxy that must actually be unbound. As such, there is a robust prediction that this should be the true correlation, and at fixed $\sigma$, systems with deeper potential wells should have higher BH masses (see Hopkins et al. 2007a). One manifestation of this is shown here - at the same $\sigma$, classical and

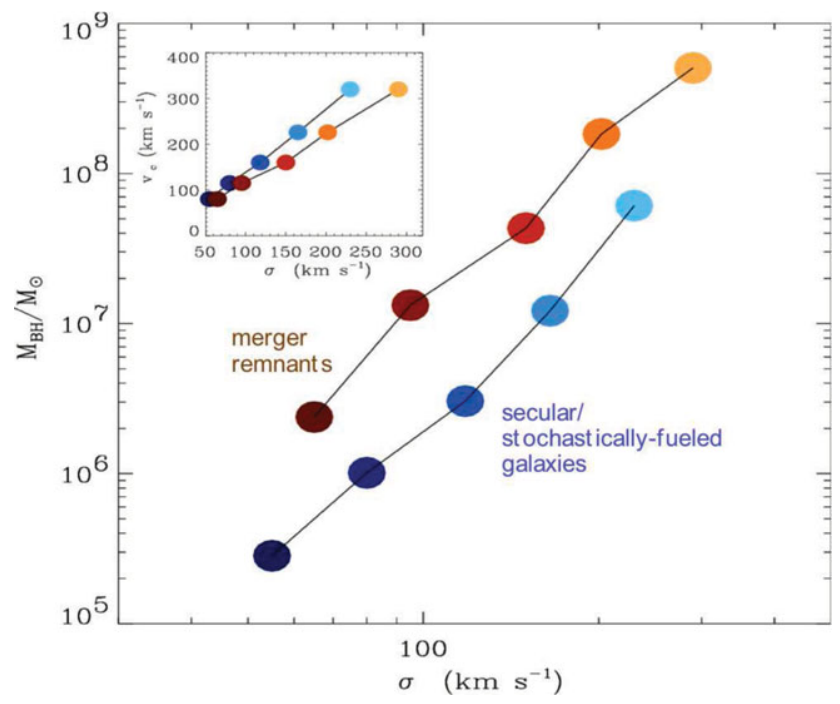

Figure 2. Prediction of feedback-regulated models such as that in Figure 1 for the different $M_{\mathrm{BH}}-\sigma$ relations in "classical" (merger remnant) and "pseudo" (secular) bulges. The two have different structural properties; hence, the relation between $\sigma$ and the quantity that actually determines BH mass in the model - the central potential well depth — is systematically different. 
pseudobulges (believed to be formed in galaxy mergers and disk instabilities, respectively) have different structural properties. The former have higher dispersions, are more isotropic, and follow $r^{1 / 4}$-law brightness profiles; the latter have more rotation, higher anisotropy, and follow exponential profiles. As such, the relation between the observed $\sigma$ and the relevant quantity, the central potential well depth, is different in the two objects. So too, in these models, should the resulting $M_{\mathrm{BH}}-\sigma$ relation differ. There may even be tentative evidence for this in the present data (Hopkins et al. 2007b; Aller \& Richstone 2007; Greene et al. 2008; Gadotti \& Kauffmann 2009).

The "blowout" induced by AGN feedback also naturally implies a long tail of declining luminosity, illustrated in Figure 3. Hopkins et al. (2005b,c) argue that this can have important implications for the AGN lifetime. Despite being an obvious over-simplification, models and inferences made from observations often assume that AGN behave as "lightbulbs," transitioning rapidly between a simple "on" state (at high Eddington ratio) and "off" state (at negligible Eddington ratio). In contrast, the models predict a continuous distribution of luminosities in time. In particular, the feedback-regulated models predict a fairly gentle transition through the blowout, especially if there is a large gas supply available for accretion. The AGN activity can persist for $\sim$ Gyr after the main episode. This has obvious implications, for example, for the abundance of AGN in transition objects between the blue cloud and red sequence. It also means that the time spent at a given luminosity is not some delta function, but a non-trivial function of that luminosity. As such, the interpretation of AGN demographics may be quite different. For example, Hopkins \& Hernquist (2009b) show that such models predict a continuous Eddington ratio distribution, with a turnover at the high and low end and power-law behavior in between, very similar to that observed (Kauffmann \& Heckman 2008).

Such non-trivial light curve or lifetime models also can have significant implications for the kinds of objects observed at different points along the AGN luminosity function. Figure 4 shows a cartoon illustration of this point. Because, as noted in Figure 3, the duration of the peak/blowout stage is short, and "triggering" events such as violent major

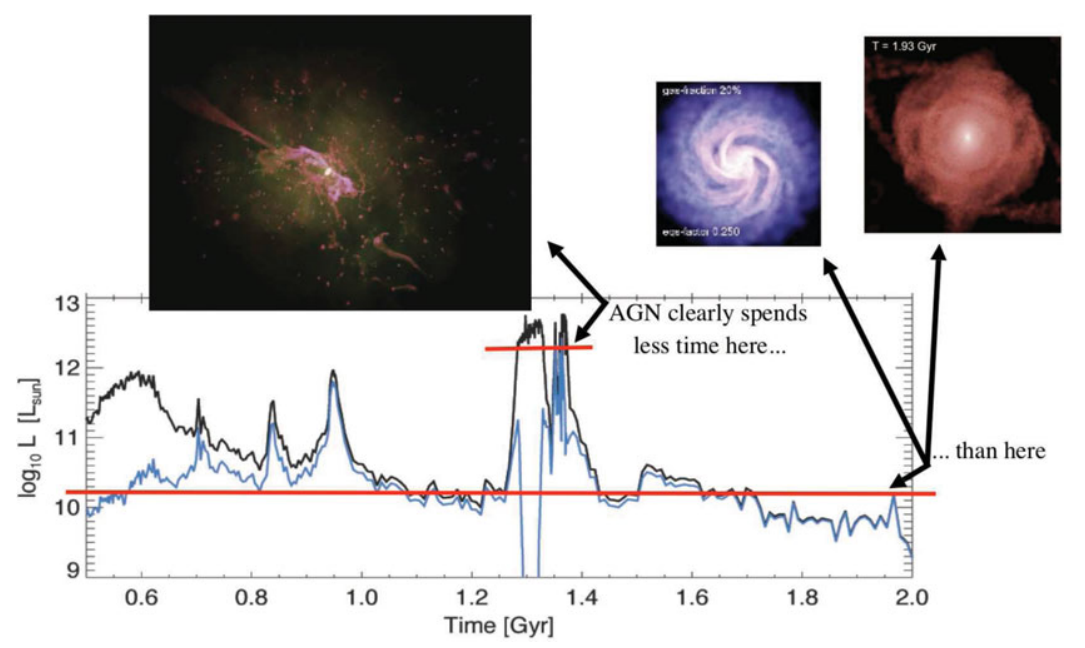

Figure 3. Implications of feedback-regulated models for AGN lifetimes. The heavy line shows the bolometric AGN luminosity in a simulated merger of two Milky Way-like disks; the lighter line shows the $B$-band luminosity after attenuation by dust. The growth-regulating "blowout" occurs at $t \approx 1.35 \mathrm{Gyr}$, and leads to a long tail of slowly declining-feedback regulated accretion. The total time spent at different luminosities is a continuous but strong function of those luminosities; as such, the AGN "lifetime" is more than just a single number. 
mergers are rare, we expect that objects caught right at this phase should be a significant fraction of the population only at the most extreme luminosities (corresponding to the brightest quasars, $M_{B} \sim-28 \mathrm{mag}$ or $M_{\mathrm{BH}} \gtrsim 10^{9} M_{\odot}$ BHs near the Eddington limit). The rapidly-rising portion of the luminosity function up to near the break at $z \sim 1-2$ $\left(M_{B} \sim-25 \mathrm{mag}\right)$ should correspond to the "post-blowout" fading/relaxing phase. In this stage, the AGN activity can continue for around a gigayear while stellar populations turn the galaxy from blue to red. Merger-induced tidal features and other obvious signatures fade very quickly — in fact in simulations they are usually not recognizable even at the peak of the blowout stage - and here are invisible in all but the deepest local observations. At lower luminosities $\left(M_{B} \sim-23 \mathrm{mag}\right)$, a mix of low-mass BHs, which will live in predominantly low-mass bulges in disk-dominated galaxies, and may be excited to accrete via a mix of stochastic processes, secular instabilities, and/or minor mergers, will become an important contribution to the population (Hopkins \& Hernquist 2006, 2009a). Also at the lowest luminosities, the truly faded, massive systems that have exhausted their gas supply will be present at very low accretion rates. The combination of dilution by their host-galaxy light (since they are at fractionally low luminosities) and the possibility that accretion becomes radiatively inefficient at such low luminosities means that the relative proportion of such objects in the low- $L$ population will be quite sensitive to the wavelengths and methods adopted (Hopkins et al. 2009a). This may naturally explain a number of observed differences in the apparent distribution of morphology, Eddington ratio, and obscured fractions between, for example, optical and X-ray selected AGN samples.

\section{Long-Term Evolution}

This may explain how black holes regulate their growth, and the associated blowout may remove gas and assist in the cessation of star formation in a short-term sense.

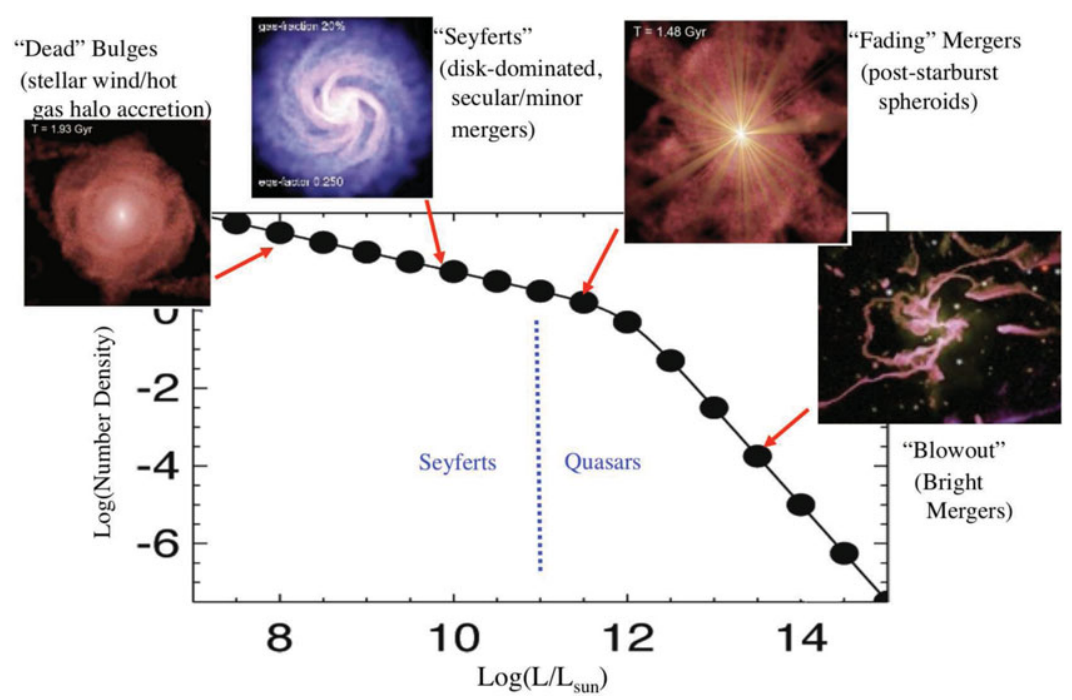

Figure 4. A proposed feedback-regulated model for the different evolutionary stages seen along the AGN luminosity function. A mix of small BHs in relatively non-violent systems (secular) and massive BHs that have had their growth shut down by feedback (dead bulges) dominate at low luminosities. Near the break, post-starburst fading spheroids, which might be transitioning from blue to red colors and be "young spheroids," but do not show the signatures of ongoing or even recent mergers (owing to rapid relaxation), dominate. At the most extreme luminosities, systems caught just at their peak or blowout stage represent an increasing fraction of the population. 
However, it does not explain how the host galaxies of massive BHs remain red forever. Especially if we think of high-redshift quasars, observations imply that the system should remain quenched for a Hubble time, despite a large amount of cold, dense gas being accreted into the halo. For this reason, AGN feedback in theoretical models is often broadly divided into two general categories, with some of their characteristics illustrated in Figure 5. The first is the "transition," "quasar," or "bright" mode of feedback. This is what we have discussed thus far - the mode associated with high-Eddington ratio growth of BHs. Because such phases are short lived, this must act rapidly. But because various arguments (e.g., Soltan 1982) imply that most of the mass in BHs is accumulated in such short-lived, high-Eddington ratio events, this must also be the mechanism that regulates the $\mathrm{BH}$ mass itself. As such, it must also act on small scales (to actually suppress accretion). To the extent that $\mathrm{BH}$ mass is correlated with the bulge mass, and not the mass of galaxy disks, then the growth of the $\mathrm{BH}$ implies - at least in a statistical sense - morphological transformation into bulge-dominated galaxies, which are also observed to be "red and dead." Therefore, this feedback mechanism should be associated with whatever process also drives the change in galaxy morphologies and the movement of mass from the blue cloud to the red sequence. It may itself assist in that movement (shutting down star formation in the short term), but the amount of gas mass that it need affect is quite small — to regulate the BH mass only $\sim 10^{-3}-10^{-2} M_{\text {gal }}$ must be expelled. Given the association with morphological change and short timescales involved, this mechanism may preferentially act in violent events such as gas-rich mergers.

On the other hand, precisely for the reasons above (e.g., the short duration of highEddington ratio activity), such feedback will not solve the cooling-flow problem and explain how red sequence galaxies remain red and dead for a Hubble time. Here, what we call "maintenance" mode (also commonly called "radio mode," as described by Croton et al. (2006), must take over. This keeps galaxies red, but need not be associated with their becoming red in the first place. Likewise, any morphological changes would be subtle
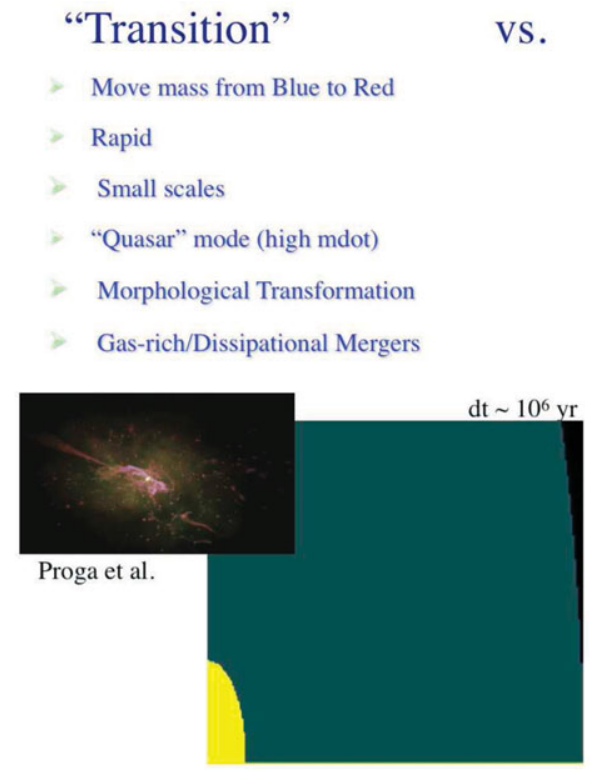

Regulates Black Hole Mass

VS.
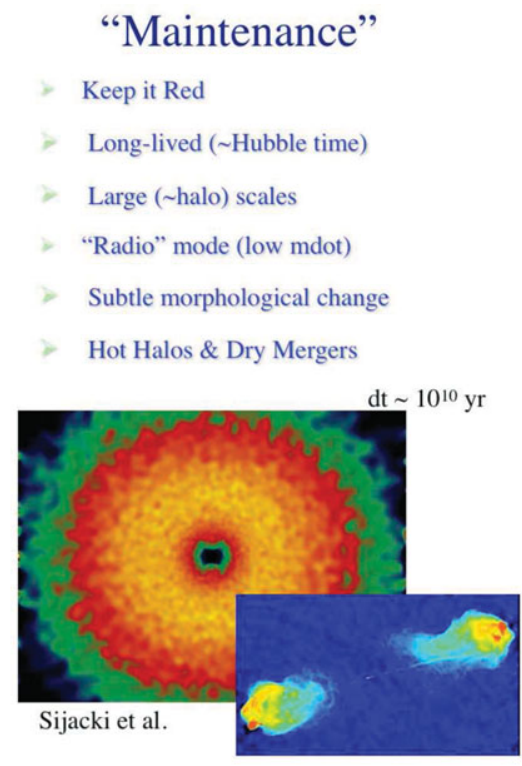

Regulates Galaxy Mass

Figure 5. Key properties of "transition" or "quasar" mode feedback versus "maintenance" or "radio" mode feedback. 
(associated with dry mergers, for example). The key is to prevent new cooling — so the feedback must act on large scales (those of the galaxy halo and cooling flow). And it must be long-lived, acting for a Hubble time. As such, it is often associated with low-luminosity AGN, perhaps driving large-scale radio jets or X-ray bubbles into the ISM. Being lowluminosity, these accretion modes are not associated with much BH growth. But because they suppress new cooling over a very large timescale, they are critical for regulating the galaxy mass. Such feedback modes are preferentially associated, in models, with massive "hot halos" wherein virial shocks establish a quasi-static atmosphere, providing a surface for the feedback to act upon (Kereš et al. 2005; Dekel \& Birnboim 2006). Whether this mode of feedback might somehow be associated with, or could interact with the immediate or after-effects of the "quasar" mode is unknown, but an important subject for future research.

\section{References}

Aller, M. C. \& Richstone, D. O. 2007, ApJ, 665, 120

Begelman, M. C. 1985, ApJ, 297, 492

Blandford, R. D. \& Payne, D. G. 1982, MNRAS, 199, 883

Crenshaw, D. M., et al. 2000, AJ, 120, 1731

Croton, D. J., et al. 2006, MNRAS, 365, 11

Dekel, A. \& Birnboim, Y. 2006, MNRAS, 368, 2

Di Matteo, T., Springel, V., \& Hernquist, L. 2005, Nature, 433, 604

Elvis, M. 2000, ApJ, 545, 63

Ferrarese, L. \& Merritt, D. 2000, ApJ, 539, L9

Gadotti, D. A. \& Kauffmann, G. 2009, MNRAS, 399, 621

Gebhardt, K., et al. 2000, ApJ, 539, L13

Graham, A. W., Erwin, P., Caon, N., \& Trujillo, I. 2001, ApJ, 563, L11

Greene, J. E., Ho, L. C., \& Barth, A. J. 2008, ApJ, 688, 159

Hall, P. B. \& Hutsemèkers, D. 2003, in Active Galactic Nuclei: From Central Engine to Host Galaxy, ed. S. Collin, F. Combes, \& I. Shlosman, (San Francisco: ASP), p. 209

Hall, P. B., Sadavoy, S. I., Hutsemèkers, D., Everett, J. E., \& Rafiee, A. 2007, ApJ, 665, 174

Hopkins, P. F. \& Elvis, M. 2009, MNRAS, in press [arXiv:0904.0649]

Hopkins, P. F. \& Hernquist, L. 2006, ApJS, 166, 1

-. 2009a, ApJ, 694, 599

-. 2009b, ApJ, 698, 1550

Hopkins, P. F., Hernquist, L., Cox, T. J., Di Matteo, T., Martini, P., Robertson, B., \& Springel, V. 2005a, ApJ, 630, 705

Hopkins, P. F., Hernquist, L., Cox, T. J., Di Matteo, T., Robertson, B., \& Springel, V. 2005b, ApJ, 630, 716

-. 2005c, ApJ, 632, 81

Hopkins, P. F., Hernquist, L., Cox, T. J., \& Kereš, D. 2008, ApJS, 175, 356

Hopkins, P. F., Hernquist, L., Cox, T. J., Robertson, B., \& Krause, E. 2007a, ApJ, 669, 45

-. 2007b, ApJ, 669, 67

Hopkins, P. F., Hernquist, L., Martini, P., Cox, T. J., Robertson, B., Di Matteo, T., \& Springel, V. 2005d, ApJ, 625, L71

Hopkins, P. F., Hickox, R., Quataert, E., \& Hernquist, L. 2009a, MNRAS, 398, 333

Hopkins, P. F., Murray, N., \& Thompson, T. A. 2009b, MNRAS, 398, 303

Hopkins, P. F., Narayan, R., \& Hernquist, L. 2006a, ApJ, 643, 641

Hopkins, P. F., Somerville, R. S., Hernquist, L., Cox, T. J., Robertson, B., \& Li, Y. 2006b, ApJ, 652,864

Johansson, P. H., Naab, T., \& Burkert, A. 2009a, ApJ, 690, 802

-. 2009b, ApJ, in press [arXiv:0910.2232]

Kauffmann, G. \& Heckman, T. M. 2008, MNRAS, in press [arXiv:0812.1224] 
Kereš, D., Katz, N., Weinberg, D. H., \& Davé, R. 2005, MNRAS, 363, 2

King, A. 2003, ApJ, 596, L27

Konigl, A. \& Kartje, J. F. 1994, ApJ, 434, 446

Laor, A., Fiore, F., Elvis, M., Wilkes, B. J., \& McDowell, J. C. 1997, ApJ, 477, 93

Lapi, A., Shankar, F., Mao, J., Granato, G. L., Silva, L., De Zotti, G., \& Danese, L. 2006, ApJ, 650,42

Magorrian, J., et al. 1998, AJ, 115, 2285

McKernan, B. \& Yaqoob, T. 1998, ApJ, 501, L29

Menci, N., Cavaliere, A., Fontana, A., Giallongo, E., Poli, F., \& Vittorini, V. 2003, ApJ, 587, L63

Miller, L., Turner, T. J., \& Reeves, J. N. 2008, A\& $A, 483,437$

Murray, N., Quataert, E., \& Thompson, T. A. 2005, ApJ, 618, 569

Proga, D. 2000, ApJ, 538, 684

-. 2007, ApJ, 661, 693

Salucci, P., Szuszkiewicz, E., Monaco, P., \& Danese, L. 1999, MNRAS, 307, 637

Sazonov, S. Y., Ostriker, J. P., Ciotti, L., \& Sunyaev, R. A. 2005, MNRAS, 358, 168

Silk, J. \& Rees, M. J. 1998, A\&A, 331, L1

Soltan, A. 1982, MNRAS, 200, 115

Somerville, R. S., Hopkins, P. F., Cox, T. J., Robertson, B. E., \& Hernquist, L. 2008, MNRAS, 391,481

Springel, V., Di Matteo, T., \& Hernquist, L. 2005, MNRAS, 361, 776

Tortora, C., Antonuccio-Delogu, V., Kaviraj, S., Silk, J., Romeo, A. D., \& Becciani, U. 2009, MNRAS, 396, 61

Tremaine, S., et al. 2002, ApJ, 574, 740

Turner, T. J., Reeves, J. N., Kraemer, S. B., \& Miller, L. 2008, A\& $A, 483,161$

Veilleux, S., Cecil, G., \& Bland-Hawthorn, J. 2005, ARAA, 43, 769

Volonteri, M., Salvaterra, R., \& Haardt, F. 2006, MNRAS, 373, 121

Weymann, R. J., Carswell, R. F., \& Smith, M. G. 1981, ARAA, 19, 41

Younger, J. D., Hopkins, P. F., Cox, T. J., \& Hernquist, L. 2008, ApJ, 686, 815

Yu, Q. \& Tremaine, S. 2002, MNRAS, 335, 965 